Pacific Journal of Mathematic 


\title{
CONVOLUTION AND SEPARATE CONTINUITY
}

\author{
JAMES C. S. Wong
}

In this paper, we extend the convolution formula

$$
\begin{aligned}
\int f d \mu * \nu & =\int f \circ \tau d \mu \times \nu=\iint f(x y) d \mu(x) d \nu(y) \\
& =\iint f(x y) d \nu(y) d \mu(x),
\end{aligned}
$$

where $f \in L_{1}(\mu * \nu), \mu, \nu \in M(S)$ and $\tau(x, y)=x y, x, y \in S$ for a locally compact group $S$ to locally compact semigroups with separately continuous multiplication. More precisely, we show that for such semigroups, the same convolution formula is valid if the measure $\mu \times \nu$ is replaced by a suitable measure on $S \times S$ (closely related to $\mu \times \nu$ ), thus improving a result of 1 . Glickberg and complementing results of $B$. E. Johnson. Some important consequences of this convolution formula in abstract harmonic analysis on separately continuous semigroups are discussed.

1. Introduction. Let $G$ be a locally compact group and $C_{0}(G)$ the continuous functions on $G$ which vanish at infinity. Let $I, J$ be nonnegative linear functionals in $C_{0}(G) *$ and $\mu, \nu$ the measures in $M(G)$ which correspond to $I, J$ respectively. The convolutions $I * J$ and $\mu * \nu$ are defined, using Riesz Representation Theorem and Fubini's Theorem by

$$
I * J(f)=\int f d \mu * \nu=\iint f(x y) d \mu(x) d \nu(y)=\iint f(x y) d \nu(y) d \mu(x),
$$

for $f \in C_{0}(G)$. Moreover, if $f \in L_{1}(\mu * \nu)$, then

$$
\begin{aligned}
\int f d \mu * \nu & =\int f \circ \tau d \mu \times \nu=\iint f(x y) d \mu(x) d \nu(y) \\
& =\iint f(x y) d \nu(y) d \mu(x),
\end{aligned}
$$

where $\tau: G \times G \rightarrow G$ is defined by $\tau(x, y)=x y$. (See Hewitt and Ross $[4, \$ 19.10]$ for details.)

If $G$ is only a locally compact semigroup with separately continuous multiplication, Glicksberg showed in [3] that the convolutions $I * J$ and $\mu * \nu$ can still be defined (without appealing to Fubini's Theorem) by the formula 


$$
I * J(f)=\int f d \mu * \nu=\iint f(x y) d \mu(x) d \nu(y)=\iint f(x y) d \nu(y) d \mu(x),
$$

if $f \in C_{0}(G)$.

In fact, it was shown that if $f \in C_{0}(G)$, then the function $y \rightarrow \int f(x y) d \mu(x)$ is bounded continuous (though not necessarily in $C_{0}(G)$ ) and the two iterated integrals always coincide (irrespective of Fubini's Theorem). However, the convolution formula $\left({ }^{*}\right)$ may or may not be valid in general.

Later, Johnson in [7] proved that if $f$ is bounded continuous (and $\tau$ separately continuous), than $f \circ \tau$ is measurable with respect to any measure in $M(G \times G)$ and therefore by Fubini's Theorem,

$$
\int f \circ \tau d \mu \times \nu=\iint f(x y) d \mu(x) d \nu(y)=\iint f(x y) d \nu(y) d \mu(x)
$$

for such $f$. In particular, the convolution formula $(*)$ is valid for any $f \in C_{0}(G)$.

In this paper, we shall prove that if $f \in L_{1}(\mu * \nu)$, then

$$
\text { (**) } \int f d \mu * \nu=\iint f(x y) d \mu(x) d \nu(y)=\iint f(x y) d \nu(y) d \mu(x),
$$

and as a consequence, the convolution formula $\left(^{*}\right)$ is true for any bounded continuous functions $f$ on $G$. The method is of course, to bypass the Fubini's Theorem and work with iterated integrals, because $f \circ \tau$ need not be measurable with respect to $\mu \times \nu$ if $f \in L_{1}(\mu * \nu)$.

It should be remarked that if multiplication in the semigroup $G$ is jointly continuous, then $f \circ \tau$ is in $L_{1}(\mu \times \nu)$ if $f$ is in $L_{1}(\mu * \nu)$ and so formula $\left({ }^{*}\right)$ is true for such $f$. The proof is actually the same as in the group case (see Hewitt and Ross [4, Theorem 19.10 p. 267], where no use is made of the continuity of inversion).

The formula $\left({ }^{* *}\right)$ is of fundamental importance in abstract harmonic analysis. It allows us to define the convolutions $\mu \odot f$ and $f \odot \mu$ of a measure $\mu \in M(G)$ and any bounded Borel function $f$. The results are generalised functions with the property that

$$
(\mu \odot f, \nu)=(f, \mu * \nu)=(f \odot \nu, \mu)
$$

where $(f, \mu)$ denotes the integral $\int f_{\mu} d \mu$ which always exists (see Wong [14] and $\$ 4$ below for details).

Many results which depend on formula $\left({ }^{* *}\right)$ but not on joint continuity of semigroup multiplication can now be carried over to separately continuous semigroups. For example, results in [10], [11], 
[12], [13] and [14] etc. This is particuląrly interesting since many semigroups are notoriously only separately continuous.

2. Notations and terminologies. For notations and terminologies in integration on locally compact spaces, we shall follow Hewitt and Ross [4]. Let $S$ be a locally compact semigroup with separately continuous multiplication and $M(S)$ the measure algebra with total variation norm and convolution $\mu * \nu$ as multiplication defined by

$$
\int f d \mu * \nu=\iint f(x y) d \mu(x) d \nu(y)=\iint f(x y) d \nu(y) d \mu(x)
$$

for $f \in C_{0}(S)$, the space of continuous functions on $S$ which vanish at infinity. (See Glicksberg [3] for details.) As in [4], we shall use the same symbol $\mu$ to denote both the outer measure and its induced measure. Note that measures in $M(S)$ are complete.

Let $\tau: S \times S \rightarrow S$ be the mapping defined by $\tau(x, y)=x y, x, y \in S$ and for each $y \in S$, define $\tau_{y}: S \rightarrow S$ and $\tau^{y}: S \rightarrow S$ by $\tau_{y}(x)=x y$ and $\tau^{y}(x)=y x, x \in S$. The maps $\tau_{y}, \tau^{y}, y \in S$ are all continuous but $\tau$ need not be so.

3. Main results. We first prove a special case of the convolution formula (**) when $f=\xi_{A}$ is the characteristic function of a $\mu * \nu$ measurable set $A$ :

$$
\mu * \nu(A)=\int \mu\left(A y^{-1}\right) d \nu(y)=\int \nu\left(x^{-1} A\right) d \mu(x),
$$

with the usual interpretation of the integrals, namely, the function $y \rightarrow \mu\left(A y^{-1}\right)$ is $|\nu|-a . e$. defined and $|\nu|-a . e$. equal to a function in $L_{1}(\nu)$, etc. Here $A y^{-1}=\{x \in S: x y \in A\}$ and similarly for $x^{-1} A$.

The proof is broken up into a series of lemmas. (See Stromberg [8] and also methods used in Hewitt and Stromberg [6, \$12.45])

LEMMA 3.1. Let $U$ be any open set in $S$ and $\mu, \nu \in M^{+}(S)$, then

$$
\mu * \nu(U)=\int \mu\left(U y^{-1}\right) d \nu(y)=\int \nu\left(x^{-1} U\right) d \mu(x) .
$$

The functions $y \rightarrow \mu\left(U y^{-1}\right)$ and $x \rightarrow \nu\left(x^{-1} U\right)$ are in fact defined everywhere and are bounded lower semicontinuous.

Proof. If $U$ is open in $S$, then $\xi_{U}$ is lower semi-continuous and hence

$$
\xi_{U}=\sup \left\{f: f \in C_{00}^{+}(S), f \leqq \xi_{U}\right\}
$$


by $[4, \S 11.10]$ where $C_{00}(S)$ are the continuous functions on $S$ with compact supports. Now for each $y \in S, \xi_{U} \circ \tau_{y}$ is also lower semicontinuous. In fact

$$
\xi_{U} \circ \tau_{y}=\sup \left\{f \circ \tau_{y}: f \in C_{00}^{+}(S), f \leqq \xi_{U}\right\}
$$

Hence by $[4, \S 11.13]$, for each $y \in S$

$$
\begin{aligned}
\int \xi_{U}(x y) d \mu(x) & =\int \xi_{U} \circ \tau_{y}(x) d \mu(x) \\
& =\sup \left\{\int f \circ \tau_{y}(x) d \mu(x): f \in C_{00}^{+}(S), f \leqq \xi_{U}\right\} \\
& =\sup \left\{\int f(x y) d \mu(x): f \in C_{00}^{+}(S), f \leqq \xi_{U}\right\}
\end{aligned}
$$

Now the function $y \rightarrow \int f(x y) d \mu(x)$ is continuous if $f \in C_{00}^{+}(S)$ by Glicksberg's result $[3, \S 1.2]$. Therefore the function $y \rightarrow$ $\int \xi_{U}(x y) d \mu(x)=\mu\left(U y^{-1}\right)$ is defined everywhere and lower semicontinuous (and obviously bounded). Moreover

$$
\begin{aligned}
\mu * \nu(U) & =\int \xi_{U} d \mu * \nu \\
& =\sup \left\{\int f d \mu * \nu: f \in C_{00}^{+}(S), f \leqq \xi_{U}\right\} \\
& =\sup \left\{\int\left(\int f(x y) d \mu(x)\right) d \nu(y): f \in C_{00}^{+}(S), f \leqq \xi_{U}\right\} \\
& =\int \sup \left\{\int f(x y) d \mu(x): f \in C_{00}^{+}(S), f \leqq \xi_{U}\right\} d \nu(y) \\
& =\iint \xi_{U}(x y) d \mu(x) d \nu(y)
\end{aligned}
$$

by applying $[4,811.13]$ again. The other half of the Lemma can be proven by considering the map $\tau^{y}$.

LEMMA 3.2. Let $F$ be $\sigma$-compact and $\mu, \nu \in M^{+}(S)$, then

$$
\mu * \nu(F)=\iint \xi_{F}(x y) d \mu(x) d \nu(y)=\iint \xi_{F}(x y) d \nu(y) d \mu(x) .
$$

The function $y \rightarrow \int \xi_{F}(x y) d \mu(x)\left(x \rightarrow \int \xi_{F}(x y) d \nu(y)\right)$ is defined everywhere and is $\nu$-measurable ( $\mu$-measurable). 
Proof. Let $F$ be compact, then $U=F^{\prime}$ is open and $\xi_{F}=1-\xi_{U}$ is then bounded measurable with respect to any measure in $M(S)$. So is $\xi_{F} \circ \tau_{y}=1-\xi_{U} \circ \tau_{y}$ for each $y \in S$. Hence

$$
y \rightarrow \int \xi_{F}(x y) d \mu(x)=\mu(X)-\int \xi_{U}(x y) d \mu(x)
$$

is defined everywhere and $\nu$-measurable by Lemma 3.1. (in fact upper semi-continuous). Moreover

$$
\begin{aligned}
\mu * \nu(F) & =\mu * \nu(X)-\mu * \nu(U) \\
& =\mu(X) \nu(X)-\iint \xi_{U}(x y) d \mu(x) d \nu(y) \\
& =\int\left\{\mu(X)-\int \xi_{U}(x y) d \mu(x)\right\} d \nu(y) \\
& =\iint \xi_{F}(x y) d \mu(x) d \nu(y) .
\end{aligned}
$$

Similarly $\mu * \nu(F)=\iint \xi_{F}(x y) d \nu(y) d \mu(x)$.

If $F$ is $\sigma$-compact, choose a sequence of compact sets $F_{n}$ such that $F_{n} \uparrow F$. Then for each $y \in S, F_{n} y^{-1} \uparrow F y^{-1}$ and by Monotone Convergence Theorem,

$$
\int \xi_{F_{n}}(x y) d \mu(x) \uparrow \int \xi_{F}(x y) d \mu(x) .
$$

Therefore $y \rightarrow \int \xi_{F}(x y) d \mu(x)$ is defined everywhere and $\nu$ measurable. Another application of Monotone Convergence Theorem gives

$\mu * \nu(F) \uparrow \mu * \nu\left(F_{n}\right)=\iint \xi_{F_{n}}(x y) d \mu(x) d \nu(y) \uparrow \iint \xi_{F}(x y) d \mu(x) d \nu(y)$.

Hence, $\mu * \nu(F)=\iint \xi_{F}(x y) d \mu(x) d \nu(y)$. Similarly $\mu * \nu(F)=$ $\iint \xi_{F}(x y) d \nu(y) d \mu(x)$.

LEMMA 3.3. Let $B$ be any subset of $S, \mu, \nu \in M^{+}(S)$, then $\mu * \nu(B) \geqq \overline{\bar{J}}_{y} \overline{\bar{I}}_{x} \xi_{B}(x y)$ and $\mu * \nu(B) \geqq \overline{\bar{I}}_{x} \overline{\bar{J}}_{y} \xi_{B}(x y)$ where $I, J$ are the non- 
negative linear functionals in $C_{0}(S)^{*}$ corresponding to $\mu, \nu$ and $\overline{\bar{I}}, \overline{\bar{J}}$ extensions of $I, J$ to nonnegative functions on $S([4, \S 11.16])$. If $\mu * \nu(B)=0$, then $\mu\left(B y^{-1}\right)=0$ for $\nu$-almost all $y \in S$ and $B y^{-1}$ is $\mu$-measurable for $\nu$-almost all $y \in S$. Similarly for $x^{-1} B$.

Proof.

$$
\begin{aligned}
\mu * \nu(B) & =\inf \{\mu * \nu(U): U \text { open, } U \supset B\} \\
& =\inf \left\{\iint \xi_{U}(x y) d \mu(x) d \nu(y): U \text { open, } U \supset B\right\} \\
& =\inf \left\{\overline{\bar{J}}_{y} \overline{\bar{I}}_{x} \xi_{U}(x y): U \text { open, } U \supset B\right\} \text { (using }[4, \S 11.36] \text { twice) } \\
& \geqq \overline{\bar{J}}_{y} \overline{\bar{I}}_{x} \xi_{B}(x y) \text { since } \xi_{U} \geqq \xi_{B}
\end{aligned}
$$

and $\xi_{U y^{-1}} \geqq \xi_{B y}{ }^{-1}$ for all $y \in S$. Similarly

$$
\mu * \nu(B) \geqq \overline{\bar{I}}_{x} \overline{\bar{J}}_{y} \xi_{B}(x y) .
$$

Now if $\mu * \nu(B)=0$, then $\overline{\bar{J}}_{y} \overline{\bar{I}}_{x} \xi_{B}(x y)=0$ and by [4, Theorem 11.27], the set $\left\{y: \mu\left(B y^{-1}\right)>0\right\}$ is $\nu$-null. Hence $B y^{-1}$ is $\mu$-null and $\mu$ measurable for $\nu$-almost all $y$. Similarly, $x^{-1} B$ is $\nu$-null and $\nu$ measurable for $\mu$-almost all $x$. (Note that the measures in $M(S)$ are complete.)

Lemma 3.4. Let $\mu, \nu \in M^{+}(S)$ and $A$ be $\mu * \nu$-measurable, then $A y^{-1}$ is $\mu$-measurable for $\nu$-almost all $y$ and $x^{-1} A$ is $\nu$-measurable for $\mu$-almost all $x$. Moreover, the function $y \rightarrow \int \xi_{A}(x y) d \mu(x)=\mu\left(A y^{-1}\right)$ is defined $\nu$-a.e. and equal' $\nu$ - a.e. to a function in $L_{1}(\nu)$. Similarly for the function $x \rightarrow \int \xi_{A}(x y) d \nu(y)=\nu\left(x^{-1} A\right)$. Moreover

$$
\mu * \nu(A)=\iint \xi_{A}(x y) d \mu(x) d \nu(y)=\iint \xi_{A}(x y) d \nu(y) d \mu(x) .
$$

Proof. Let $A \subset S$ be $\mu * \nu$-measurable. Write $A=B \cup F$ where $F$ is $\sigma$-compact and $B$ is $\mu * \nu$-null, $B \cap F=\varnothing$. For each $y, A y^{-1}=$ $B y^{-1} \cup F y^{-1}$ and $\xi_{A y^{-1}}=\xi_{B y^{-1}}+\xi_{F y^{-1}}$. Now $\xi_{F y-1}$ is $\mu$-measurable and by Lemma 3.3, $B y^{-1}$ is $\mu$-measurable and $\mu$-null for $\nu$-almost all $y$. Hence $A y^{-1}$ is $\mu$-measurable and $\mu\left(A y^{-1}\right)=\mu\left(F y^{-1}\right)$ for $\nu$-almost all $y$. Consequently, the function $y \rightarrow \int \xi_{A}(x y) d \mu(x)=\mu\left(A y^{-1}\right)$ is defined $\nu$-a.e. and is $\nu$-a.e. equal to $\int \xi_{F}(x y) d \mu(x)=\mu\left(F y^{-1}\right)$ which is in $L_{1}(\nu)$. Moreover 


$$
\begin{aligned}
\mu * \nu(A) & =\mu * \nu(F) \\
& =\iint \xi_{F}(x y) d \mu(x) d \nu(y) \\
& =\iint \xi_{A}(x y) d \mu(x) d \nu(y) .
\end{aligned}
$$

A similar argument completes the proof of the Lemma.

THEOREM 3.5. If $\mu, \nu \in M^{+}(S), f \in L_{1}(\mu * \nu)$, then

$$
\int f d \mu * \nu=\iint f(x y) d \mu(x) d \nu(y)=\iint f(x y) d \nu(y) d \mu(x)
$$

with the usual interpretation of iterated integrals.

Proof. This follows immediately from Lemma 3.4 using standard convergence arguments.

Corollary 3.8. Let $f \in L_{1}(\mu * \nu)$ and suppose that $f$ is also measurable with respect to the $\sigma$-ring $\mathscr{B}_{1}$ generated by the compact $G_{\delta}$ 's in $S$. Then $f \circ \tau$ is $\mu \times \nu$-measurable and

$$
\begin{aligned}
\int f d \mu * \nu & =\int f \circ \tau d \mu \times \nu=\iint f(x y) d \mu(x) d \nu(y) \\
& =\iint f(x y) d \nu(y) d \mu(x) .
\end{aligned}
$$

Proof. It is enough to show that $f \circ \tau$ is $\mu \times \nu$-measurable. The corollary then follows from Theorem 3.5 and Fubini's Theorem. Since the map $\tau: S \times S \rightarrow S$ is separately continuous, by a result of Johnson [7, Theorem 2.2], for each $B \in \mathscr{B}_{1}, \tau^{-1}(B)$ is measurable with respect to any measure in $M(S \times S)$. But $f$ is $\mathscr{B}_{1}$-measurable. Hence $f \circ \tau$ is $\mu \times \nu$ measurable.

REmarks. The sets in $\mathscr{B}_{1}$ are sometimes called the Baire sets (e.g. in [1]). However, we follow [4] or [6] and define the Baire sets as the $\sigma$-ring $\mathscr{B}_{0}$ generated by the sets of the form $G=\{x: f(x)>0\}$ or equivalently sets of the form $Z=\{x: f(x)=0\}$ where $f$ is continuous. $\mathscr{B}_{0}$ contains all compact $G_{\delta}$ 's and hence $\mathscr{B}_{1}$. It is wellknown that if $S$ is compact, each $Z$ is a compact $G_{\delta}$ and $\mathscr{B}_{0}=\mathscr{B}_{1}$. If $S$ is compact metric, then $\mathscr{B}_{0}=\mathscr{B}_{1}=\mathscr{B}$ the Borel sets.

Consequently, if $S$ is compact and $f \in L_{1}(\mu * \nu)$ is also Baire measurable, then $f \circ \tau$ is $\mu \times \nu$-measurable and the convolution formula $\left({ }^{*}\right)$ holds for such $f$. (See also $[2, \S 1.2]$.) 
It should be pointed out that there are plenty of compact separately continuous semigroups. For example, the weakly almost periodic compactification of any separately continuous semigroup (not necessarily locally compact).

It is also interesting to observe that although $f \circ \tau$ need not be $\mu \times \nu$-measurable if $f \in L_{1}(\mu * \nu)$ and $\tau$ only separately continuous, there is always a measure $\mu \otimes \nu$ on $S \times S$ such that $f \circ \tau$ is $\mu \otimes \nu$ measurable when $f \in L_{1}(\mu * \nu)$. Moreover $f \circ \tau \in L_{1}(\mu \otimes \nu)$ and

$$
\begin{aligned}
\int f d \mu * \nu & =\int f \circ \tau d \mu \otimes \nu=\iint f(x y) d \mu(x) d \nu(y) \\
& =\iint f(x y) d \nu(y) d \mu(x) .
\end{aligned}
$$

The measure $\mu \otimes \nu$ is defined as follows: Let $\mathscr{S}=\left\{\tau^{-1}(A): A\right.$ is $\mu * \nu$ measurable\}. Then $\mathscr{S}$ is a $\sigma$-algebra. We define $\mu * \nu$ on $\mathscr{S}$ by putting

$$
\mu \otimes \nu\left(\tau^{-1}(A)\right)=\mu * \nu(A)
$$

where $A$ is $\mu * \nu$ measurable. This is well defined. For if $\tau^{-1}(A)=$ $\tau^{-1}(B)$ with $A, B \mu * \nu$ measurable, then $x y \in A$ iff $x y \in B$ for any $x, y \in S$. (If $S$ has identity, then $A=B$ and there is nothing to prove.) Therefore, $A y^{-1}=B y^{-1}$ for all $y \in S$. By Lemma 3.4,

$$
\mu\left(A y^{-1}\right)=\mu\left(B y^{-1}\right)
$$

for $\nu$-almost all $y$ and

$$
\mu * \nu(A)=\int \mu\left(A y^{-1}\right) d \nu(y)=\int \mu\left(B y^{-1}\right) d \nu(y)=\mu * \nu(B) .
$$

Now it is straightforward to show that $\mu \otimes \nu$ is a finite measure on $\mathscr{S}$ (though not necessarily in $M(S \times S)$ ). The very definition of $\mu \otimes \nu$ gives the convolution formula $\left({ }^{* *}\right)$ when $f$ is the characteristic function and a standard (e.g. monotone convergence) argument establishes the same formula for $f \in L_{1}(\mu * \nu)$.

The $\mu * \nu$ measurable sets and the sets in $\mathscr{S}$ in general may not be the same but they both contain the $\sigma$-ring $\tau^{-1}\left(\mathscr{B}_{1}\right)$. Consequently, if $f \in L_{1}(\mu * \nu)$ is also $\mathscr{B}_{1}$ measurable, then $f \circ \tau$ is both $\mu \times \nu$ and $\mu \otimes \nu$ measurable and

$$
\int f \circ \tau d \mu \times \nu=\int f \circ \tau d \mu \otimes \nu
$$


In particular, the same equality obtains for $f \in C_{00}(S)$ (see also [1, §56.1]). Of course if $\tau$ is jointly continuous, then each set in $\mathscr{S}$ is $\mu \times \nu$ measurable and $\mu \times \nu$ agrees with $\mu \otimes \nu$ on $\mathscr{S}$.

4. Comments. The convolution formula

$$
\int f d \mu * \nu=\iint f(x y) d \mu(x) d \nu(y)=\iint f(x y) d \nu(y) d \mu(x),
$$

$f \in L_{1}(\mu * \nu), \mu, \nu \in M(S)$ has some far reaching consequences in abstract harmonic analysis for separately continuous semigroups.

First, it can be used to show that the probability measures on $S$ form a semigroup (algebraically) under convolution. Moreover, their supports or carriers satisfy the condition

$$
\text { carrier } \mu * \nu=[\text { carrier } \mu \cdot \operatorname{carrier} \nu]^{-}, \mu, \nu \in M_{0}(S) \text {. }
$$

These are extensions of results in Glicksberg [3, \$4] for compact separately continuous semigroups (in which case the probability measures on $\boldsymbol{S}$ form even a compact separately continuous semigroup under convolution and weak* topology of $\left.M(S)=C_{0}(S)^{*}\right)$. Since the constant functions are in $L_{1}(\mu * \nu)$, it follows from the convolution formula above that the convolution of two probability measures is again a probability measure. To prove the assertion about their carriers, we argue as follows: Let $A=$ carrier $\mu$ and $B=$ carrier $\nu$. Then $C=(A B)^{-}$is closed, hence $\mu * \nu$ measurable. If we apply the convolution formula to the function $f=\xi_{C}$, the characteristic function of $C$, noting that $\xi_{A}(x) \xi_{B}(y) \leqq \xi_{C}(x y)$ for all $x, y \in S$, we have

$$
\begin{gathered}
1 \geqq \mu * \nu(C)=\int \xi_{C} d \mu * \nu=\iint \xi_{C}(x y) d \mu(x) d \nu(y) \\
\geqq \iint \xi_{A}(x) \xi_{B}(y) d \mu(x) d \nu(y)=\mu(A) \nu(B)=1 .
\end{gathered}
$$

To show that any open set $W$ which intersects $C$ has $\mu * \nu(W)>0$, the arguments used in the proof in Glicksberg [3, Lemma 4.1] can be repeated here because $S$ is locally compact (Hausdorff) hence completely regular.

Second, many results which depend on this convolution formula but not on joint continuity of semigroup multiplication can now be established for locally compact separately continuous semigroups. For examples, results in Wong [10], [11], [12], [13] and [14]. Each time the Fubini's Theorem is called for the equality of the integrals $\int f d \mu * \nu$, 
$\iint f(x y) d \mu(y) d \nu(y)$ and $\iint f(x y) d \nu(y) d \mu(x)$ in the jointly continuous case, it is now ensured by the convolution formula in the separately continuous situation as long as $f \in L_{1}(\mu * \nu)$. As a case in point, we can now define the convolution $\mu \odot f$ (and $f \odot \mu$ ) of a measure $\mu \in M(S)$ and a generalised function $f \in G L(S)$ where $f=\left(f_{\mu}\right)_{\mu \in M(S)} \in$ $\Pi\left\{L_{\infty}(|\mu|): \mu \in M(S)\right\}$ is called a generalised function if $f_{\mu}=f_{\nu}|\mu|-$ a.e. whenever $\mu \ll \nu$ and $\|f\|=\sup \left\{\left\|f_{\mu}\right\|_{\mu, \infty}\|\mu\| \leqq 1\right\}<\infty$. It is known that the Banach space $G L(S)$ is isometrically isomorphic to $M(S)^{*}$ and that $G L(S)$ contains all bounded (Borel) measurable functions (see Wong [14] for more details and results in this direction).

If $f \in G L(S), \mu \in M(S), \mu \odot f$ is defined by

$$
(\mu \odot f)_{\nu}=\mu \odot f_{\mu * \nu}, \nu \in M(S),
$$

where $\mu \odot f_{\mu * \nu}(y)=\int f_{\mu * \nu}(x y) d \mu(x)$ for $|\nu|$-almost all $y$ in $S . \quad \mu \odot f_{\mu * \nu}$ determines unambiguously an equivalence class in $L_{\propto}(|\nu|)$. Moreover, $\mu \odot f$ is again a generalised function and we have the identity

$$
(\mu \odot f, \nu)=(f, \mu * \nu)=(f \odot \nu, \mu) .
$$

Here $f \odot \nu$ is defined similarly and $(f, \mu)=\int f_{\mu} d \mu$ if $f \in G L(S)$ and $\mu \in M(S)$.

All these follow from the Fubini's Theorem (and measurability of $f \circ \tau$ ) in the jointly continuous case but are now obtainable from our convolution formula in the separately continuous situation.

It is interesting to observe that if $S$ is a locally compact group and $f \in L_{\infty}(S)$ is also Borel measurable, then $\mu \odot f$ is just $\mu^{\sim} * f$ where $\mu^{\sim} \in M(S)$ is defined by $\mu^{\sim}(E)=\mu\left(E^{-1}\right)$ and of course we identify a bounded Borel measurable function $f$ with the generalised function $\left(f_{\mu}\right)_{\mu \in M(s)}$ such that $f_{\mu}=f$ for all $\mu \in M(S)$. For $f \odot \nu$, the result is less elegant (because of the distinction between left and right Haar measures) under this identification. In fact $f \odot \nu$ corresponds to $\Delta\left((1 / \Delta) f * \nu^{\sim}\right)$ where $\Delta$ is the modular function and the above identity becomes

$$
\left(\mu^{\sim} * f, \nu\right)=(f, \mu * \nu)=\left(\Delta\left(\frac{1}{\Delta} f * \nu^{\sim}\right), \mu\right)
$$

where $(f, \mu)=\int f d \mu$. For unimodular groups, this is

$$
\left(\mu^{\sim} * f, \nu\right)=(f, \mu * \nu)=\left(f * \nu^{\sim}, \mu\right) .
$$


In general, if $f$ is bounded Borel measurable, $\mu, \nu \in M_{\alpha}(S)$, the absolutely continuous measures in $M(S)$ (with respect to a fixed left Haar measure $\lambda$ ), the identity then reads

$$
\left(\frac{1}{\Delta} \phi^{\sim} * f, \psi\right)=(f, \phi * \psi)=\left(f * \psi^{\sim}, \phi\right),
$$

where $\phi, \psi$ are the functions in $L_{1}(S)=M_{\alpha}(S)$ corresponding to $\mu$ and $\nu$, $(f, \phi)=\int f \phi d \lambda$ and $\phi^{\sim}(x)=\phi\left(x^{-1}\right), x \in S$. (See Hewitt and Ross [4, $\$ 20.12, \S 20.13$ and $\$ 20.14]$ and Hewitt and Ross [5, Addendum to Volume I, p. 726].)

\section{REFERENCES}

1. S. K. Berberian, Measure and integration, MacMillan, New York, 1965.

2. I. Glicksberg, Convolution semigroups of measures, Pacific J. Math., 9 (1959), 51-67.

3. _- Weak Compactness and separate continuity, Pacific J. Math., 11 (1961), 205-214.

4. E. Hewitt and K. A. Ross, Abstract harmonic analysis I, Springer-Verlag, Berlin, 1963.

5. —-, Abstract harmonic analysis II, Springer-Verlag, New York, 1970.

6. E. Hewitt and K. Stromberg, Real and abstract analysis, Springer-Verlag, New York, 1969.

7. B. E. Johnson, Separate continuity and measurability, Proc. Amer. Math. Soc., 20 (1969), 420-422.

8. K. Stromberg, A note on the convolution of regular measures, Math. Scand., 7 (1959), 347-352.

9. J. Williamson, Harmonic analysis on semigroup, J. London Math. Soc., 42 (1967), 1-41.

10. J. C. S. Wong, Invariant means on locally compact semigroups, Proc. Amer. Math. Soc., 31 (1972), 39-45.

11. - An ergodic property of locally compact amenable semigroups, Pacific J. Math., 48 (1973), 615-619.

12. _ Absolute continuous measures on locally compact semigroups, Canad. Math. Bull., 18 (1975), 127-131.

13. - A characterisation of topological left thick subsets in locally compact left amenable semigroups, Pacific J. Math., 62 (1976), 295-303.

14. - Abstract harmonic analysis of generalised functions on locally compact semigroups with application to invariant means, to appear in Australian J. Math.

Received May 16, 1977. Research supported by the National Research Council of Canada Grant No. A8227.

UNIVERSITY OF CALGARY

Calgary, Canada T2N 1N4 



\section{PACIFIC JOURNAL OF MATHEMATICS}

\section{EDITORS}

RICHARD ARENS (Managing Editor)

University of California

Los Angeles, CA 90024

R. A. BeAumont

University of Washington

Seattle, WA 98105

C. C. MOORE

University of California

Berkeley, CA 94720
J. DUGUNDJI

Department of Mathematics

University of Southern California

Los Angeles, CA 90007

R. FINN AND J. MILGRAM

Stanford University

Stanford, CA 94305

\section{ASSOCIATE EDITORS}
E. F. BECKENBACH
B. H. NEUMANN
F. WOLF
K. YoshidA

\section{SUPPORTING INSTITUTIONS}

UNIVERSITY OF BRITISH COLUMBIA

UNIVERSITY OF SOUTHERN CALIFORNIA

CALIFORNIA INSTITUTE OF TECHNOLOGY

STANFORD UNIVERSITY

UNIVERSITY OF CALIFORNIA

UNIVERSITY OF HAWAII

MONTANA STATE UNIVERSITY

UNIVERSITY OF TOKYO

UNIVERSITY OF NEVADA

UNIVERSITY OF UTAH

NEW MEXICO STATE UNIVERSITY

OREGON STATE UNIVERSITY

UNIVERSITY OF OREGON

OSAKA UNIVERSITY

WASHINGTON STATE UNIVERSITY

UNIVERSITY OF WASHINGTON

AMERICAN MATHEMATICAL SOCIETY

The Supporting Institutions listed above contribute to the cost of publication of this Journal, but they are not owners or publishers and have no responsibility for its contents or policies.

Mathematical papers intended for publication in the Pacific Journal of Mathematics should be in typed form or offset-reproduced (not dittoed), double spaced with large margins. Underline Greek letters in red, German in green, and script in blue. The first paragraph or two must be capable of being used separately as a synopsis of the entire paper. Items of the bibliography should not be cited there unless absolutely necessary, in which case they must be identified by author and Journal, rather than by item number. Manuscripts, in duplicate, may be sent to any one of the four editors. Please classify according to the scheme of Math. Reviews, Index to Vol. 39. All other communications should be addressed to the managing editor, or Elaine Barth, University of California, Los Angeles, California, 90024.

100 reprints are provided free for each article, only if page charges have been substantially paid. Additional copies may be obtained at cost in multiples of 50 .

The Pacific Journal of Mathematics is issued monthly as of January 1966. Regular subscription rate: $\$ 72.00$ a year (6 Vols., 12 issues). Special rate: $\$ 36.00$ a year to individual members of supporting institutions.

Subscriptions, orders for numbers issued in the last three calendar years, and changes of address should be sent to Pacific Journal of Mathematics, 103 Highland Boulevard, Berkeley, California, 94708.

PUBLISHED BY PACIFIC JOURNAL OF MATHEMATICS, A NON-PROFIT CORPORATION

Printed at Jerusalem Academic Press, POB 2390, Jerusalem, Israel. 


\section{Pacific Journal of Mathematics}

\section{Vol. 75, No. $2 \quad$ October, 1978}

Susan Jane Zimmerman Andima and W. J. Thron, Order-induced

topological properties ................................... 297

Gregory Wade Bell, Cohomology of degree 1 and 2 of the Suzuki groups . . 319

Richard Body and Roy Rene Douglas, Rational homotopy and unique

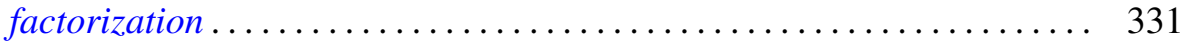

Frank Lewis Capobianco, Fixed sets of involutions ................. 339

L. Carlitz, Some theorems on generalized Dedekind-Rademacher sums .... 347

Mary Rodriguez Embry and Alan Leslie Lambert, The structure of a special class of weighted translation semigroups .....................

Steve Ferry, Strongly regular mappings with compact ANR fibers are

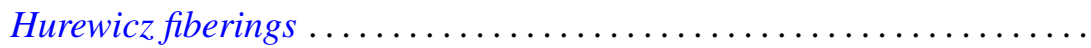

Ivan Filippenko and Marvin David Marcus, On the unitary invariance of the

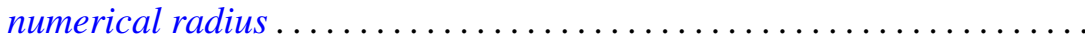

$\mathrm{H}$. Groemer, On the extension of additive functionals on classes of convex

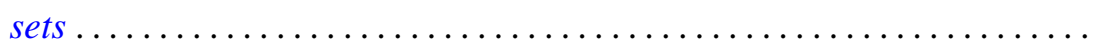

Rita Hall, On the cohomology of Kuga's fiber variety ............... 411

H. B. Hamilton, Congruences on $\mathrm{N}$-semigroups ................. 423

Manfred Herrmann and Rolf Schmidt, Regular sequences and lifting

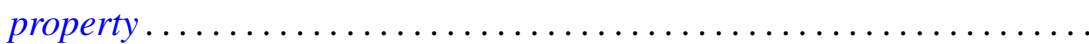

James Edgar Keesling, Decompositions of the Stone-Čech compactification which are shape equivalences .....................

Michael Jay Klass and Lawrence Edward Myers, On stopping rules and the expected supremum of $S_{n} / T_{n}$

Ronald Charles Linton, $\lambda$-large subgroups of $C_{\lambda}$-groups

William Owen Murray, IV and L. Bruce Treybig, Triangulations with the free cell property ............................

Louis Jackson Ratliff, Jr., Polynomial rings and $H_{i}$-local rings ...

Michael Rich, On alternate rings and their attached Jordan rings....

Gary Sampson and H. Tuy, Fourier transforms and their Lipschitz

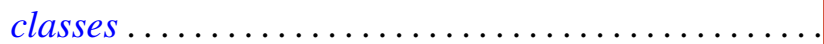

Helga Schirmer, Effluent and noneffluent fixed points on dendrites ...

Daniel Byron Shapiro, Intersections of the space of skew-symmetric maps

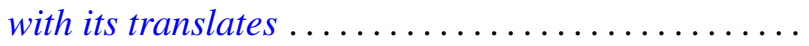

Edwin Spanier, Tautness for Alexander-Spanier cohomology ...

Alan Stein and Ivan Ernest Stux, A mean value theorem for binary digits ...

Franklin D. Tall, Normal subspaces of the density topology . .

William Yslas Vélez, Prime ideal decomposition in $F\left(\mu^{1 / p}\right) \ldots$ 